\title{
DISEÑO Y VALIDACIÓN DE UNA ESTRATEGIA PARA ENSEÑANZA-APRENDIZAJE METACOGNITIVO DE HABILIDADES PARA PROFESORES DE EDUCACIÓN PRIMARIA ${ }^{1}$
}

\author{
DESIGN AND VALIDATION OF A STRATEGY FOR \\ METACOGNITIV EDUCATION-LEARNING OF SKILLS \\ FOR TEACHERS OF PRIMARY EDUCATION
}

Verónica Gutiérrez Soriano Julio Herminio Pimienta Prieto

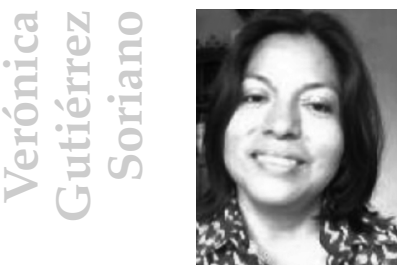

Doctora en Educación: «Diagnóstico, medida y evaluación de la intervención educativa». Profesora de la Escuela Normal La Paz de Veracruz. Coordinadora de la instrumentación, implementación y sistematización de la práctica docente en condiciones reales de trabajo de las Licenciaturas en Educación Especial, Preescolar y Primaria. Correo electrónico:

[vgutierrez@cpv.edu.mx].

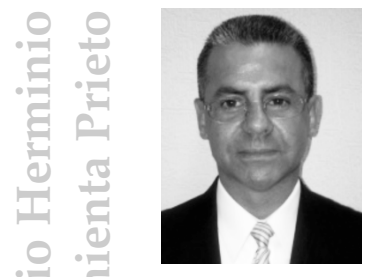

Doctor en Educación: «Diagnóstico, medida y evaluación de la intervención educativa». Profesor investigador de la Universidad Anáhuac, México-Norte. Coordinador del Programa Doctoral «Medida y evaluación de la intervención educativa», en la misma universidad. Líneas de investigación: evaluación de los aprendizajes y evaluación de intervenciones educativas. Correo electrónico: [julio.pimienta@anahuac.mx]; [juliopi@prodigy.net.mx].

\section{RESUMEN}

La investigación tuvo como propósito proponer una Estrategia de Enseñanza Aprendizaje Metacognitivo de Habilidades para Profesores de Educación Primaria (EEAMHPP), realizar un acercamiento a la validación de su puesta en práctica, e indagar las perspectivas

\footnotetext{
Esta investigación es producto de la tesis doctoral presentada por Verónica Gutiérrez Soriano (septiembre, 2010), en la Facultad de Educación de la Universidad Anáhuac, México-Norte, misma que fue guiada por el doctor Pimienta Prieto.
} 
de enseñanza y aprendizaje de un grupo de profesores formadores de docentes. EEAMHPP se fundamenta en concepciones del aprendizaje humano de la perspectiva constructivista y considera un enfoque integral de la personalidad en relación con la formación de competencias (J. Piaget, 1977; Vygotsky, 1988; Ausubel, 1976; Bruner, 1990; Coll, 1990; Carretero, 1997; Bandura, 1999; Gimeno y Pérez, 1992; Castellanos, 2002; Perrenoud, 2004; Pimienta, 2010; Saint-Onge, 1997; Jackson, 2000; Rodríguez y Bermúdez, 1996; Moltó, 2001; Pimienta, 2006). Se implementaron dos paradigmas en investigación: cuantitativo, a través de un diseño cuasiexperimental y cualitativo, al considerar fundamentos de la etnografía educativa.

Los resultados registran un incremento significativo del rendimiento académico de los estudiantes del grupo experimental con respecto del grupo control ( $\left.{ }^{*} \mathrm{p}<.05\right)$. Los docentes que realizaron la intervención educativa obtuvieron un incremento significativo en la evaluación del desempeño docente con respecto a los que continuaron con la utilización del enfoque didáctico tradicional ( $\left.{ }^{*} \mathrm{p}<.05\right)$.

Palabras clave: aprendizaje humano; constructivismo; estrategia de enseñanza y aprendizaje metacognitivo; desarrollo integral de la personalidad; formación docente.

\section{ABSTRACT}

The aim of this investigation was to suggest a Learning-Teaching Strategy of Metacognitive Skills for Primary Teachers (EEAMHPP ${ }^{2}$ ), to perform an approach to the validation of its practice and to inquire into teaching and learning perspectives of a group of instructors training teachers. The strategy is based on human learning concepts from the constructivist point of view and considers a comprehensive approach of personality in relation with the training of skills. (Piaget, J. 1977; Vygotsky,1988; Ausubel, 1976; Bruner, 1990; Coll, 1990; Carretero,

2 By its acronym in Spanish. 
1997; Bandura, 1999; Gimeno y Pérez, 1992; Castellanos, 2002; Perrenoud, 2004; Pimienta, 2010), (Saint-Onge, 1997; Jackson, 2000; Rodríguez y Bermúdez, 1996; Moltó, 2001; Pimienta, 2006). Two major research paradigms have been implemented: quantitative, through a quasi-experimental and qualitative outline, pondering the fundamentals of educational ethnography.

The results reflect a significant increase of students academic performance in the experimental group in relation to those in the control group ( $\left.{ }^{*} \mathrm{p}<.05\right)$. Teachers who carried out an educational intervention gained meaningful knowledge in the assessment achievement with regard to those who continued using the didactic traditional proposal $\left({ }^{*} \mathrm{p}<.05\right)$.

Key words: human learning; constructivism; teaching strategies and metacognitive learning; comprehensive approach of personality; teacher's training.

\section{INTRODUCCIÓN}

El proceso de enseñanza aprendizaje se concibe como el objeto principal de estudio de la Didáctica, el cual, por sus características, es bastante complejo. Precisamente, este proceso transcurre mediante un sistema de actuaciones de educadores y educandos, para que estos últimos se apropien de aquella parte de la cultura humana necesaria para adaptarse a vivir en su contexto de actuación y así transformarlo (Danilov y Skatkin, 1980; Moltó, 2001).

No son ajenas las dificultades que enfrentan algunos profesores en su práctica docente, particularmente al querer dar seguimiento al proceso de enseñanza aprendizaje e identificar las relaciones que guardan los componentes del diseño didáctico entre sí, y estos, con el docente y el discente. Se resalta, tan solo en el ámbito didáctico, el desconocimiento del saber procedimental, cómo enseñarlo y cómo evaluarlo (SEP, 2003). 
Por ello, hoy ante la implementación de propuestas curriculares basadas en competencias para la educación, cobra relevancia trascendental identificar, diseñar y valorar los componentes esenciales del proceso enseñanza aprendizaje. La formación que se exige a los docentes es para actuar como: «agentes fundamentales de la intervención educativa, los maestros son los verdaderos agentes del desarrollo curricular», señala este documento rector (SEP, 2008a, p. 32-33).

Por tanto,

[...] es imperativa la creación y difusión de alternativas que contribuyan a mover el estado imperante de las cuestiones educativas. Urge un movimiento que propicie la reflexión acerca de la práctica docente; pero que genere la producción de propuestas factibles de llevar a cabo dentro del salón de clases (Pimienta, 2006, p. IV).

Lo anterior permite repensar las acciones educativas que surgen ante el perfil de egreso de docentes — prioritariamente de educación primaria- que demanda, entre un conjunto de rasgos que es necesario promover, los siguientes: desarrollo y consolidación de habilidades intelectuales básicas; dominio de los contenidos de la enseñanza en educación primaria y competencias didácticas para la enseñanza de los contenidos. Otro aspecto importante que debe caracterizar al profesor es la identidad profesional y ética con su profesión. Finalmente, los docentes han de apropiarse de las competencias necesarias para valorar y respetar la diversidad regional, social, cultural y étnica de nuestro país (SEP, 1997).

En este trabajo se ha retomado la formación de habilidades en los estudiantes a través del proceso educativo, en particular las generales, que constituyen el dominio del significado y de una secuencia de acciones, que es un aspecto deficiente. En esto influyen, entre otros, los aspectos siguientes: en el campo de la didáctica, los investigadores han prestado más atención a la formación de los conocimientos, que a la formación de las habilidades; no ha existido un entendimiento suficiente de la naturaleza psíquica diferente de los conocimientos y las habilidades, lo cual ha llevado a la utilización de formas similares para 
la enseñanza y el aprendizaje de ambos objetos psíquicos; existe un dominio no suficiente, por parte de los educadores, acerca de los aspectos cognitivos e instrumentales relacionados con estas habilidades, y se observa ausencia de metodologías concretas para su enseñanza (Rodríguez y Bermúdez, 1996 y Moltó, 2001; Pimienta, 2011).

El estudio realizado llevó a precisar, también, que estas habilidades deben ser aprendidas por el estudiante de manera significativa, como se plantea en los documentos normativos para la formación de este profesional, y de manera metacognitiva. Se entiende como aprendizaje metacognitivo de una habilidad: el dominio de un procedimiento y su significado con flexibilidad, autorregulación y solidez. El aprendizaje metacognitivo se entiende como la apropiación que hace el estudiante de una habilidad de forma autorregulada, es decir, que sea consciente de cómo ha aprendido, pudiendo lograr su autoevaluación; además, que sea capaz de plantear lo aprendido en diferentes objetos, situaciones de los mismos y/o contextos, es decir, con flexibilidad, y un tiempo después (no menor de un mes) que se dé por logrado este aprendizaje, o sea, con solidez (Moltó, 2001).

En la práctica, toda habilidad se encuentra formando parte generalmente de una competencia, entendiéndose por competencia:

[...] la actuación integral ante actividades y problemas del contexto, con idoneidad y ética, integrando el saber ser, hacer y conocer en una perspectiva de mejora continua (Tobón, Pimienta y García, 2010, p. 11).

Ejemplifiquemos: el observar una clase o bien el observar a través de un microscopio, son dos competencias diferentes, pero ambas contienen la habilidad observar. Por tanto, si la persona no posee la habilidad de observar, no podrá lograr estas competencias.

El estudio teórico realizado permitió precisar como fundamentos de la Estrategia de Enseñanza Aprendizaje Metacognitivo, los postulados de la Perspectiva Constructivista desde un enfoque integral de la personalidad y en relación con la formación de competencias (Piaget, J. 1977; Vygotsky,1988; Ausubel, 1976; Flavell, 1978; Bruner, 1990; Coll, 
1990; Gimeno y Pérez, 1992; Rodríguez y Bermúdez, 1996; Carretero, 1997; Saint-Onge, 1997; Bandura, 1999; Jackson, 2000; Moltó, 2001; Castellanos, 2002; Perrenoud, 2004, Pimienta, 2006; Pimienta, 2010).

La concepción de estrategia que sustenta este trabajo, es el

[...] uso deliberado y planificado de una secuencia compuesta por acciones o procedimientos dirigida a alcanzar una meta establecida [...]. Existen estrategias para alcanzar un objetivo a corto plazo y otras que requerirán el despliegue sostenido de acciones más complejas, pues son para alcanzar resultados a más largo plazo (Pozo, 1998, p. 300).

Desde esta perspectiva, se plantea que existen estrategias con diferentes niveles de generalidad, lo cual trae como consecuencia que, dentro de una estrategia más general, puedan existir estrategias particulares. Lo planteado es lo que sucede en nuestra propuesta.

En la estrategia se perciben las siguientes etapas:

1. Propedéutica o preactiva (Saint-Onge, 1997; Gutiérrez y Moltó, 2004, y Gutiérrez y Moltó, 2007).

2. Activa, compuesta por las siguientes fases didácticas: introducción, desarrollo, sistematización y consolidación (Saint-Onge, 1997; Gutiérrez y Moltó, 2001, y Gutiérrez y Moltó, 2007).

3. Post activa (Saint-Onge, 1997; Gutiérrez y Moltó, 2004, y Gutiérrez y Moltó, 2007).

En el planteamiento de la estrategia subyace un sistema, porque cada etapa se entiende en relación con las otras y no solo en sí misma. Por tanto, cada una se realiza por ser necesaria al sistema. A continuación se analizan las etapas de la estrategia que constituyen procedimientos sistemáticos que favorecen un proceso de enseñanza aprendizaje de apropiación, tanto en lo cognitivo como en lo instrumental.

En la etapa propedéutica o preactiva, de acuerdo con Moltó (2001), la característica esencial es la de preparar las condiciones para comenzar el aprendizaje de la habilidad en cuestión. Por tanto, se requiere 
comprender la necesidad de organizar la creación del diseño didáctico, la mediación a partir del diseño de tareas; además de la programación de la distribución a los miembros del grupo en las distintas tareas prediseñadas. En sí, esta etapa constituye la planeación de la clase en su conjunto, es decir, las decisiones que prevé el docente al realizar el bosquejo de la secuencia de tareas didácticas y donde es consciente de los elementos que subyacen en estas, tales como:

- Los propósitos u objetivos.

- Los contenidos de enseñanza y aprendizaje que han de ser posesión de los alumnos (habilidades, conocimientos y valores morales), y la interrelación entre ellos. En el caso de las habilidades, debe tenerse bien claro el significado de las mismas y su sistema de acciones componentes.

- La forma en que se van a llevar a cabo la enseñanza y el aprendizaje, o sea, el sistema de tareas educativas. En el caso de las habilidades, el sistema debe ir garantizando el aprendizaje metacognitivo de las mismas.

- Los recursos o medios didácticos que se utilizarán en el proceso del sistema diseñado para la sesión.

- La evaluación, la forma en que se realizará la misma durante la clase y los tipos que serán necesarios llevar a cabo.

En esta etapa es necesario prever cómo se establecerán los vínculos en cada tarea, entre las tareas y en cada fase. Es prioritario definir: la familiarización del sujeto con el objeto a aprender; identificar el nivel de dominio con lo nuevo a aprender; planteamiento de tareas didácticas guiadas; valoración del dominio que el estudiante va alcanzando; trabajo con apoyos externos; verbalización de lo aprendido de forma oral y escrita, y evaluación de lo aprendido a partir de tareas sin apoyo externo. Esta idea es interesante, porque no basta con plantear los contenidos de enseñanza adecuadamente, sino que es básico saber cómo, qué y cuándo hacer, para lograr que los vínculos logren eficazmente su propósito. De acuerdo a la experiencia obtenida, estos son fundamentales en la implementación de las tareas (Moltó, 2001). 
Al realizar lo anterior, también es importante distribuir en el tiempo programado las tareas diseñadas. Es prudente seleccionar los recursos didácticos pertinentes y el diseño de instrumentos de registro que permitirán al profesor llevar un seguimiento de la realización, por parte de los alumnos, de las tareas previstas y de los avances obtenidos en su aprendizaje en cada fase de la etapa activa. Por último, en esta etapa preactiva es sustancial, antes de cada actuación, revisar las tareas destinadas a cada momento de la interacción con los alumnos y registrar los resultados obtenidos en los instrumentos previstos que dan seguimiento a la apropiación que van teniendo los educandos.

En la etapa activa, los docentes han de dirigir el proceso de las fases didácticas de introducción, desarrollo, sistematización y consolidación, porque es el momento en que surge la interacción con los alumnos y dirigen su aprendizaje. Los enseñantes, han de conocer en cada fase didáctica ya señalada, la calidad de la construcción que realiza cada futuro profesor para la apropiación de la habilidad en el contenido del aprendizaje concreto. Lograr lo anterior con los estudiantes es una tarea ardua, pues requiere de la ejecución de una secuencia de tareas apropiadas, para que aprendan a aprender y a enseñar.

La introducción permite valorar el nivel de preparación del estudiante para el aprendizaje de la habilidad dada. Es decir, establecer el diagnóstico, para evidenciar el dominio que tiene el alumno del significado y del sistema de acciones de la habilidad que debe aprender. También debe diagnosticarse el dominio que posee de los conocimientos e instrumentaciones previas relacionadas con la habilidad a aprender. Para ello, es importante adjudicarle tareas didácticas previstas que permitirán evidenciar su nivel de dominio. Identificado lo anterior, es preciso aplicar tareas apropiadas para la eliminación de sus deficiencias cognitivas, o bien orientar o fortalecer si existe alguna noción de la habilidad.

En esta concepción del proceso de enseñanza aprendizaje, el estudiante debe diagnosticarse integralmente, no solo en lo cognitivo e instrumental, sino en aspectos tales como su situación social, salud, aptitudes, principales motivaciones, etcétera. Es por ello que en esta 
etapa de introducción no lo señalamos como parte de, pero consideramos que debe tomarse en cuenta, y de no existir, el profesor debe realizar las gestiones necesarias para obtener la mencionada información. Además, es preciso no descartar los vínculos mencionados anteriormente en cada tarea, entre las tareas de cada fase de esta etapa.

En un momento dado, el docente ha de registrar, en los instrumentos elaborados para ello, el resultado que van teniendo los estudiantes al realizar tareas didácticas en esta fase diagnóstica.

El profesor valorará a los educandos y verificará si las tareas previamente planificadas son las adecuadas para continuar el proceso de la clase, o habrá que ajustarlas o rediseñarlas a las necesidades del momento. Desde el diagnóstico, es importante identificar a los alumnos con claras ventajas, a quienes poseen regular ventaja y a los estudiantes de deficientes ventajas, para decidir la organización en equipos de trabajo de los miembros del grupo de la clase. Se recomienda que esta distribución sea de cuatro, de tres o de dos personas, hasta llegar a la realización de tareas de forma individual. Es preciso que el profesor vaya tomando todas estas decisiones pero haciendo partícipe a los alumnos, para que estén conscientes de su formación.

El Desarrollo se caracteriza porque el profesor guía al alumno en las tareas didácticas iniciales. Se hace necesario guiar paso a paso a los alumnos con ejemplos claros y precisos que lo lleven a una actuación autónoma posterior. Enseguida, se requerirá al estudiante a partir de la actuación en su equipo; se trata de tareas didácticas específicas que lo llevan al dominio de las habilidades en contenidos concretos de aprendizaje.

Estos equipos de estudiantes establecerán acuerdos para exponer el resultado de su tarea, de forma escrita u oral. En cada exposición, propondrán cuál es el significado y el sistema de acciones aprendidos, resultando en un contenido concreto de enseñanza y aprendizaje. Lo anterior se analizará grupalmente, pero tomando en cuenta el parecer de cada miembro del equipo con el que trabajaron cada tarea. 
Cada equipo autoevaluará o coevaluará su trabajo según el instrumento de registro que permita valorar la apropiación que se va alcanzando, para concienciar su nivel de avance. Es preciso que el profesor permanezca en el aula - atento a la interacción del equipo y del grupo- para poder valorar, específica y generalmente, a los estudiantes, al ir haciendo los registros evaluativos de las tareas didácticas y de seguimiento del trabajo en la clase que ellos van desarrollando. De esta forma, al revisar los datos no le parecerán ajenos, pues tendrá el referente del contacto personal.

Los alumnos escribirán en su cuaderno el resultado obtenido acerca del sistema de acciones y el significado de la habilidad en contenidos concretos. Es conveniente que, al inicio del proceso de enseñanza aprendizaje y al conformar los equipos, el profesor haya seleccionado a los responsables de los mismos (responsable del grupo o alumno monitor); esto permitirá que el educador pueda multiplicarse en la atención a los estudiantes.

En el Desarrollo, el estudiante debe aprender: el significado de la habilidad; alcanzar el conocimiento del sistema de acciones componentes, y cómo aprende él de forma particular el contenido concreto de enseñanza y aprendizaje. Esta valoración es importante para una formación educativa sólida. Lo anterior es significativo si se le permite, además, reflexionar sobre su actuación para mejorarla, de tal manera que lo que aprende le sirva para un desempeño consciente en el contexto social en el que interactúa e interactuará.

Por lo general, los estudiantes no llegan a dominar totalmente la ejecución con la reflexión acerca del sistema de acciones componentes pero, al menos, se espera sean conscientes de que determinados procesos tienen procedimientos generales que deben dominar para una correcta ejecución. Sin embargo, no se descarta que algunos dominen todas las acciones.

En esta fase, mientras los miembros del equipo no sean conscientes del significado y los procedimientos asociados con la habilidad 
que trabajan en contenidos concretos de enseñanza aprendizaje —en las distintas interacciones que realizarán a partir de las tareas indicadas-, podrán contar con un apoyo externo: cuaderno de trabajo, libro de texto, materiales didácticos e, incluso, la ayuda de un estudiante o el mismo profesor.

Al mismo tiempo, es importante que el docente enfatice al grupo lo valioso de la independencia en el aprendizaje. Es indispensable que el estudiante alcance su independencia cognoscitiva mediante una correcta dependencia de su profesor y la toma de conciencia de su estado en cuanto a esta independencia.

La sistematización y consolidación, es una fase concebida para que los educandos consoliden y sistematicen lo aprendido a partir de la realización de tareas. A ella arribarán los estudiantes, con diferentes niveles de desempeño en cada acción componente $\mathrm{y}$, por tanto, no es necesario que realicen las mismas tareas. Es pertinente llevar a cabo tareas educativas en parejas y de manera individual.

Al organizar las parejas es recomendable que el docente esté atento a ubicar alumnos aventajados en el aprendizaje con aquellos que poseen menos ventajas. Así, no solo los más aventajados ayudan a los menos aventajados, sino que los primeros desarrollan valores morales como el respeto, la solidaridad y responsabilidad. La concepción anterior se destaca porque si el apoyo externo llega al estudiante de un igual, le es más efectivo. Además, se considera que, en la medida que el alumno más aventajado ayuda a otro, va adquiriendo experiencias relacionadas con la enseñanza que resultan fundamentales en quien pretende aprender a aprender.

Debe quedar claro que, en cada momento, debe hacerse un uso adecuado de las tareas en clase. En esta fase se mantiene el trabajo con apoyo externo y la verbalización para aquellos estudiantes que aún lo necesiten. Es importante que el profesor registre el desempeño de cada estudiante y asigne tareas extra clase acordes a las necesidades de cada alumno. 
En la etapa post-activa se toman decisiones acerca del aprendizaje alcanzado por los estudiantes, para tener objetividad en la designación de las tareas extra clase y prever el diseño de la próxima sesión (Saint-Onge, 1997 y Moltó, 2001).

Aunque la evaluación siempre está presente en cada una de las etapas anteriores - preactiva o propedéutica, y activa-, en esta etapa, la evaluación es final y lleva a decidir sobre el futuro accionar del profesor con cada estudiante en sesiones posteriores. Las hojas de evaluación o instrumentos de registros han de plantear los aspectos a tener en cuenta durante la valoración que se vaya haciendo en cada tarea desarrollada en las etapas anteriores. Es preciso que, en estos instrumentos, puedan apreciarse explícitamente las acciones componentes en contenidos concretos de aprendizaje, lo mismo que una escala para valorar el grado de dominio alcanzado en ellas por los estudiantes.

Durante todo el proceso, la evaluación valora los conocimientos previos y el dominio del significado y del sistema de acciones de los estudiantes. Para ello, el seguimiento evaluativo mostrará el despliegue en la ejecución con cada acción del sistema de la habilidad que se trabaja; el profesor podrá apreciarlo al revisar los registros del seguimiento realizado durante las fases de la etapa activa.

Las tareas asignadas a los educandos, después de la valoración de cada fase, les permiten aplicar el procedimiento asociado con la habilidad en un contenido de aprendizaje concreto, con una frecuencia y periodicidad relacionadas con la complejidad del mismo y, también, las dificultades detectadas durante el aprendizaje. Además, debe lograrse flexibilidad en esta aplicación para emplearse en situaciones diferentes. La etapa post-activa permite al profesor autoevaluar su desempeño en la dirección del proceso de aprendizaje de sus estudiantes para prever la siguiente sesión de clase.

Según Rodríguez y Bermúdez (1996), las principales características de la evaluación del aprendizaje metacognitvo de habilidades, pueden plantearse en una escala sintética para dar seguimiento al avance 
de la apropiación. En el estudio se propuso dar seguimiento al proceso a partir de rúbricas —estableciendo la dimensión, el nivel de logro y el criterio que delimitan gradualmente los procedimientos a alcanzar-, diario de trabajo y portafolio de información.

Lo planteado hasta aquí permite destacar que, en la lógica actual de la educación en el país, se olvidó casi en su totalidad, la función y perspectivas de la Didáctica General además, de las Didácticas Específicas. La actividad se ha centrado en los diseños y evaluaciones curriculares, pasando por alto el aporte imprescindible de la Didáctica en la formación de los docentes (Díaz Barriga, 1997). Es en este punto donde esta investigación adquiere un mayor sentido e importancia, dentro de los estudios ya realizados en México.

La situación problemática precisada en este estudio es el no alcance del perfil de egreso del plan de estudios 1997, por parte de los futuros licenciados en Educación Primaria, en particular en el campo de desarrollo de habilidades intelectuales, reportado como suficiente a nivel estatal. Esto último, se aprecia en los resultados en los años: 2005, 2007 y 2009, realizada por el Centro Nacional de Evaluación (CENEVAL), a través de la prueba EGEL (Examen General de Egreso) y emitidos por la Secretaría de Educación Pública (SEP). Esto particularmente surge en el desarrollo de habilidades como observar, identificar, caracterizar, comparar y clasificar, las que por su carácter de básicas son esenciales, pues permiten la apropiación de la mayor parte de los conocimientos, están presentes en los sistemas de acciones de otras habilidades y forman parte de una gran cantidad de competencias generales y profesionales.

El estudio realizado corroboró esta situación. Esta problemática, después de 13 años de la implementación del plan de estudios 1997, requiere de propuestas viables y factibles que permitan la apropiación de estas habilidades por parte de los futuros docentes de educación primaria.

Lo planteado permitió esbozar el problema de la investigación a través del siguiente cuestionamiento: ¿Será posible lograr un 
aprendizaje metacognitivo, de las habilidades mencionadas de los estudiantes de la Licenciatura en Educación Primaria, e incrementar la percepción del desempeño académico por parte de los estudiantes?

\section{OBJETIVOS GENERALES DE LA INVESTIGACIÓN:}

- Diseñar una estrategia para la enseñanza y el aprendizaje metacognitivo de las habilidades: observar, identificar, caracterizar, comparar y clasificar; en la formación de estudiantes de la Licenciatura en Educación Primaria, basada fundamentalmente en postulados de la Perspectiva Constructivista, desde un enfoque integral de la personalidad y en relación con la formación de competencias.

- Realizar un primer acercamiento a la validación de la estrategia diseñada.

- Indagar acerca de las perspectivas de la enseñanza y el aprendizaje, que sustentan la práctica docente de un grupo de profesores, formadores de docentes de educación primaria, de escuelas normales privadas del Estado de Veracruz.

\section{HIPÓTESIS Y VARIABLES DE LA INVESTIGACIÓN}

En correspondencia con los objetivos anteriormente expuestos, las hipótesis planteadas fueron:

Hipótesis 1: Los alumnos de la Licenciatura en Educación Primaria, intervenidos con la estrategia diseñada (grupo experimental), lograrán un aprendizaje metacognitivo significativamente superior de las habilidades, que el otro grupo de alumnos de la Licenciatura en Educación Primaria que siguen trabajando con la estrategia tradicional de enseñanza (grupo control). 
Variables:

a) Variable independiente: estrategia de enseñanza aprendizaje metacognitivo de habilidades para estudiantes de la Licenciatura de Educación Primaria.

b) Variable dependiente: aprendizaje metacognitivo de las habilidades observar, identificar, caracterizar, comparar y clasificar.

Hipótesis 2: Los profesores de los estudiantes que participan en la intervención educativa, aplicando la estrategia diseñada, lograrán un desempeño docente significativamente superior que los docentes que continúan trabajando con la estrategia tradicional.

Variables:

a) Variable independiente: estrategia de enseñanza aprendizaje metacognitivo de habilidades para estudiantes de la Licenciatura de Educación Primaria.

b) Variable dependiente: desempeño docente.

En este trabajo, por razones propias del estudio realizado, se considera el desempeño docente como:

[...] la evaluación que realizan los estudiantes de sus profesores, recogida esta información por medio de un instrumento que ha sido construido para este efecto (Pimienta, J., 2006, p. 164).

\section{METODOLOGÍA}

Muestra. En el Estado de Veracruz, las escuelas normales privadas poseen una población de 1,878 estudiantes y 265 docentes. Las escuelas que participaron en el estudio cuentan con una población de 1,178 estudiantes y 124 docentes. De estas últimas, incluidas como parte de nuestra muestra intencional, existen 546 estudiantes asignados en 18 grupos de forma aleatoria, 9 grupos para el grupo control y 9 grupos para el grupo experimental, haciendo un total de 273 estudiantes para 
el grupo control y 273 para el grupo experimental. Finalmente, se capacitaron en la estrategia a 83 docentes, y de estos, fueron seleccionados 9 para el grupo experimental, atendiendo a la disponibilidad que mostraron. De los profesores no capacitados, se consultó con 9 para su participación en el grupo control, mismos que aceptaron. En el estudio cualitativo participaron 4 profesores del grupo experimental como informantes claves e igual número en el grupo control.

Planificación de la investigación. Se definió como una investigación evaluativa mixta, desde los enfoques cuantitativo y cualitativo, desarrollada en dos fases: cuantitativa y cualitativa. En la fase cuantitativa, el tipo de estudio aplicado fue cuasi experimental con dos grupos no equivalentes, llamados también grupos intactos: experimental y control. Es importante considerar aquí que, en la validación realizada, tuvo que optarse por la alternativa de elegir un diseño de grupo de control no equivalente. Este diseño fue elegido, dado que se buscaba someter a experimentación una estrategia de enseñanza y aprendizaje, en tanto era necesario adaptarse a las disposiciones de autoridades educativas que tienen ya una organización de los grupos y, desde luego, no permiten que aulas enteras sean perturbadas durante el trabajo o divididas para proporcionar muestras equivalentes o aleatorias, o simplemente no permiten que si lo que se prueba «es bueno», lo reciban solo algunos (Campbell y Stanley, 1991).

El mencionado diseño se realizó como sigue: se evaluó de entrada a ambos grupos y posteriormente se aplicó la estrategia solo al grupo de estudiantes de la Licenciatura en Educación Primaria (grupo experimental). Al final, se evaluó a los dos grupos de estudio, es decir, tanto al experimental, como a los estudiantes de la Licenciatura de Educación Primaria, que no habían recibido el mencionado tratamiento educativo (grupo control); asimismo, se evaluó el desempeño de los docentes participantes en el estudio. De esta forma se percibió la realización de los dos primeros objetivos. En una segunda fase, en el enfoque cualitativo, se empleó la metodología de estudio de casos con indagación fenomenológica para el alcance del tercer objetivo. 
Análisis y aplicación del instrumento de obtención de datos. Se utilizaron dos instrumentos: una prueba — elaborada para tal efectopara medir el aprendizaje metacognitivo de las habilidades: observar, identificar, caracterizar, comparar y clasificar, y una escala Likert - también diseñada para los propósitos de la investigación- para medir el desempeño docente.

Atendiendo al análisis de la confiabilidad para el primero, se realizó utilizando el método Alfa de Cronbach obteniendo un valor de 0.90, misma que podemos considerar como buena. La media de aciertos obtenida puede advertir un nivel bajo de respuestas correctas, lo que pudiera apreciarse como problemas en el grado de dificultad que representan para los sujetos algunos ítems. El resultado de la correlación Biserial Puntual (0.27), sugiere también dificultades en algunos reactivos, particularmente en el nivel de dificultad. En cuanto a la validez de contenido, se realizó mediante el juicio de 9 expertos. En el análisis de los ítems de inicio, se aprecia que en cuanto al nivel de facilidad, de forma general, la mayoría de los ítems tienen una puntuación debajo de $(\mathrm{p}>0.85)$ y por arriba de $(\mathrm{p}<0.15)$ que de acuerdo a lo que establece Seock-Ho (1999), se estiman índices de facilidad intermedia. Para el análisis de los ítems se utilizó la correlación Biserial Puntual la que resultó en que la mayor parte de los ítems pueden considerarse como razonablemente buenos. Se aprecia también que la mayoría de las opciones correctas tienen correlaciones con resultados positivos.

En cuanto a la escala Likert se realizó también un análisis global del instrumento, atendiendo a la confiabilidad como consistencia interna, utilizando el método Alfa de Cronbach, obteniendo un valor de 0.96 . Posteriormente se elaboró un análisis de confiabilidad considerando la partición por mitades, se obtuvo un Alfa de Cronbach de 0,902 para la parte 1 y de 0.954 para la parte 2 , mismas que se aprecian correlacionadas. Atendiendo a lo anterior, es posible plantear que el instrumento posee una buena confiabilidad. Este instrumento, empleado para medir el desempeño docente, ha sido tomado de Pimienta (2006), con la aclaración de que las preguntas abiertas no fueron utilizadas en este momento por el interés de 
nuestra investigación. En el análisis de los ítems, se observa en la correlación ítem-total, corregida, que oscilan entre 0,20 y 0,82 concluyendo que son adecuadas.

Los instrumentos mencionados se aplicaron en formato impreso, situado cada estudiante en su aula de clases.

Análisis de los resultados. Se realizó, primeramente, un estudio exploratorio con la finalidad de detectar problemas de casos faltantes, sesgos en la estimación de los parámetros de tendencia central y desviaciones de la normalidad.

En el estudio exploratorio no se revelaron casos faltantes y en los sesgos en la estimación de los parámetros de tendencia central se aprecia una variabilidad no significativa. Respecto a los casos desviados y la desigualdad de varianza, se estima que los casos desviados fueron mínimos y solamente se observaron algunas desigualdades de varianza en el postest.

\section{Tabla1. Resumen del análisis exploratorio de datos.}

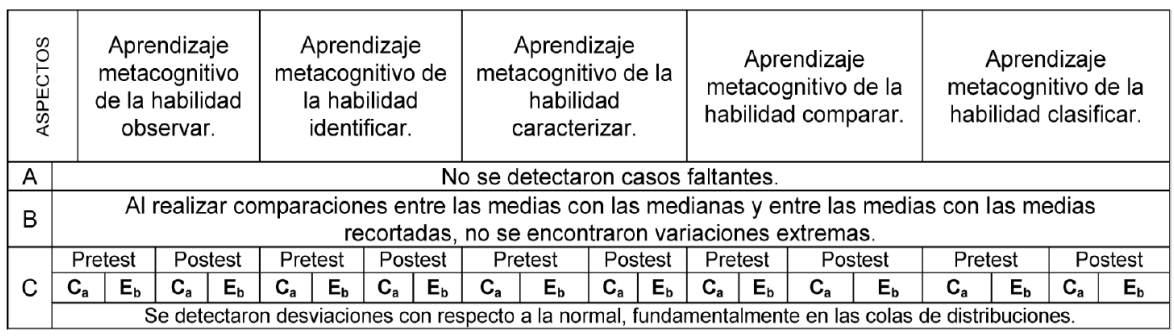

Posteriormente se elaboró un estudio de diferencias significativas, empleando la prueba $t$. 
Tabla 2. Estadísticos de grupo, para el aprendizaje metacognitivo de las habilidades observar, identificar, caracterizar, comparar, y clasificar antes y después de la intervención.

Group Statistics

\begin{tabular}{|ll|r|r|r|r|}
\hline & Grupo & N & Mean & Std. Deviation & $\begin{array}{c}\text { Std. Error } \\
\text { Mean }\end{array}$ \\
\hline pre-observar & Control & 273 & 4.08 & 1.901 & .115 \\
& Experimental & 273 & 4.07 & 1.905 & .115 \\
\hline pos-observar & Control & 273 & 4.85 & 2.633 & .159 \\
& Experimental & 273 & 7.94 & 3.153 & .191 \\
\hline pre-identificar & Control & 273 & 2.65 & .772 & .047 \\
& Experimental & 273 & 2.53 & .772 & .047 \\
\hline pos-identificar & Control & 273 & 3.75 & 1.906 & .115 \\
& Experimental & 273 & 5.60 & 1.919 & .116 \\
\hline pre-caracterizar & Control & 273 & 3.28 & .922 & .056 \\
& Experimental & 273 & 3.41 & 1.046 & .063 \\
\hline pos-caracterizar & Control & 273 & 4.36 & 1.766 & .107 \\
& Experimental & 273 & 6.84 & 2.303 & .139 \\
\hline pre-comparar & Control & 273 & 2.95 & 1.572 & .095 \\
& Experimental & 273 & 2.75 & 1.286 & .078 \\
\hline pos-Comparar & Control & 273 & 2.62 & 1.313 & .079 \\
& Experimental & 273 & 5.26 & 2.142 & .130 \\
\hline pre-clasificar & Control & 273 & 3.54 & 1.534 & .093 \\
& Experimental & 273 & 3.55 & 1.472 & .089 \\
\hline pos-clasificar & Control & 273 & 4.99 & 1.490 & .090 \\
& Experimental & 273 & 6.09 & 2.106 & .127 \\
\hline
\end{tabular}


Tabla 3. Prueba t muestras independientes para las variables intervinientes antes y después de la intervención, considerando el grupo control y experimental.

\begin{tabular}{|c|c|c|c|c|c|c|c|c|c|c|}
\hline \multicolumn{11}{|c|}{ Independent Samples Test } \\
\hline & & \multicolumn{2}{|c|}{$\begin{array}{l}\text { Levene's Test for } \\
\text { Equality of Variances }\end{array}$} & \multicolumn{7}{|c|}{ t-test for Equality of Means } \\
\hline & & \multirow[b]{2}{*}{$\mathrm{F}$} & \multirow[b]{2}{*}{ Sig. } & \multirow[b]{2}{*}{$t$} & \multirow[b]{2}{*}{ df } & \multirow[b]{2}{*}{ Sig. (2-tailed) } & \multirow{2}{*}{$\begin{array}{c}\text { Mean } \\
\text { Difference }\end{array}$} & \multirow{2}{*}{$\begin{array}{l}\text { Std. Error } \\
\text { Difference }\end{array}$} & \multicolumn{2}{|c|}{$\begin{array}{l}95 \% \text { Confidence } \\
\text { Interval of the } \\
\text { Difference }\end{array}$} \\
\hline & & & & & & & & & Lower & Upper \\
\hline pre-observar & $\begin{array}{l}\text { Equal variances } \\
\text { assumed } \\
\text { Equal variances } \\
\text { not assumed }\end{array}$ & .044 & .835 & $\begin{array}{l}.112 \\
.112\end{array}$ & $\begin{array}{r}544 \\
543.998\end{array}$ & $\begin{array}{l}.911 \\
.911\end{array}$ & $\begin{array}{l}.018 \\
.018\end{array}$ & $\begin{array}{l}.163 \\
.163\end{array}$ & $\begin{array}{l}-.302 \\
-.302\end{array}$ & $\begin{array}{l}.338 \\
.338\end{array}$ \\
\hline pos-observar & $\begin{array}{l}\text { Equal variances } \\
\text { assumed } \\
\text { Equal variances } \\
\text { not assumed }\end{array}$ & 11.480 & .001 & $\begin{array}{l}-12.436 \\
-12.436\end{array}$ & $\begin{array}{r}544 \\
527.235\end{array}$ & $\begin{array}{l}.000 \\
.000\end{array}$ & $\begin{array}{l}-3.092 \\
-3.092\end{array}$ & $\begin{array}{l}.249 \\
.249\end{array}$ & $\begin{array}{l}-3.580 \\
-3.580\end{array}$ & $\begin{array}{l}-2.603 \\
-2.603\end{array}$ \\
\hline pre-identificar & $\begin{array}{l}\text { Equal variances } \\
\text { assumed } \\
\text { Equal variances } \\
\text { not assumed }\end{array}$ & .259 & .611 & $\begin{array}{l}1.719 \\
1.719\end{array}$ & $\begin{array}{r}544 \\
543.999\end{array}$ & $\begin{array}{l}.086 \\
.086\end{array}$ & $\begin{array}{l}.114 \\
.114\end{array}$ & $\begin{array}{l}.066 \\
.066\end{array}$ & $\begin{array}{l}-.016 \\
-.016\end{array}$ & $\begin{array}{l}.243 \\
.243\end{array}$ \\
\hline pos-identificar & $\begin{array}{l}\text { Equal variances } \\
\text { assumed } \\
\text { Equal variances } \\
\text { not assumed }\end{array}$ & .347 & .556 & $\begin{array}{l}-11.254 \\
-11.254\end{array}$ & $\begin{array}{r}544 \\
543.976\end{array}$ & $\begin{array}{l}.000 \\
.000\end{array}$ & $\begin{array}{l}-1.842 \\
-1.842\end{array}$ & $\begin{array}{l}.164 \\
.164\end{array}$ & $\begin{array}{l}-2.164 \\
-2.164\end{array}$ & $\begin{array}{r}-1.521 \\
-1.521\end{array}$ \\
\hline pre-caracterizar & $\begin{array}{l}\text { Equal variances } \\
\text { assumed } \\
\text { Equal variances } \\
\text { not assumed }\end{array}$ & 5.388 & .021 & $\begin{array}{r}-1.475 \\
-1.475\end{array}$ & $\begin{array}{r}544 \\
535.520\end{array}$ & $\begin{array}{l}.141 \\
.141\end{array}$ & $\begin{array}{l}-.125 \\
-.125\end{array}$ & $\begin{array}{l}.084 \\
.084\end{array}$ & $\begin{array}{l}-.290 \\
-.290\end{array}$ & $\begin{array}{l}.041 \\
.041\end{array}$ \\
\hline pos-caracterizar & $\begin{array}{l}\text { Equal variances } \\
\text { assumed } \\
\text { Equal variances } \\
\text { not assumed }\end{array}$ & 15.106 & .000 & $\begin{array}{l}-14.138 \\
-14.138\end{array}$ & $\begin{array}{r}544 \\
509.706\end{array}$ & $\begin{array}{l}.000 \\
.000\end{array}$ & $\begin{array}{l}-2.484 \\
-2.484\end{array}$ & $\begin{array}{l}.176 \\
.176\end{array}$ & $\begin{array}{l}-2.829 \\
-2.829\end{array}$ & $\begin{array}{l}-2.138 \\
-2.138\end{array}$ \\
\hline pre-comparar & $\begin{array}{l}\text { Equal variances } \\
\text { assumed } \\
\text { Equal variances } \\
\text { not assumed }\end{array}$ & .086 & .769 & $\begin{array}{l}1.669 \\
1.669\end{array}$ & $\begin{array}{r}544 \\
523.357\end{array}$ & $\begin{array}{l}.096 \\
.096\end{array}$ & $\begin{array}{l}.205 \\
.205\end{array}$ & $\begin{array}{l}.123 \\
.123\end{array}$ & $\begin{array}{l}-.036 \\
-.036\end{array}$ & $\begin{array}{l}.447 \\
.447\end{array}$ \\
\hline pos-comparar & $\begin{array}{l}\text { Equal variances } \\
\text { assumed } \\
\text { Equal variances } \\
\text { not assumed }\end{array}$ & 50.136 & .000 & $\begin{array}{r}-17.370 \\
-17.370\end{array}$ & $\begin{array}{r}544 \\
451.021\end{array}$ & $\begin{array}{l}.000 \\
.000\end{array}$ & $\begin{array}{l}-2.641 \\
-2.641\end{array}$ & $\begin{array}{l}.152 \\
.152\end{array}$ & $\begin{array}{l}-2.940 \\
-2.940\end{array}$ & $\begin{array}{l}-2.342 \\
-2.342\end{array}$ \\
\hline pre-clasificar & $\begin{array}{l}\text { Equal variances } \\
\text { assumed } \\
\text { Equal variances } \\
\text { not assumed }\end{array}$ & .120 & .730 & $\begin{array}{l}-.085 \\
-.085\end{array}$ & $\begin{array}{r}544 \\
543.092\end{array}$ & $\begin{array}{l}.932 \\
.932\end{array}$ & $\begin{array}{l}-.011 \\
-.011\end{array}$ & $\begin{array}{l}.129 \\
.129\end{array}$ & $\begin{array}{l}-.264 \\
-.264\end{array}$ & $\begin{array}{l}.242 \\
.242\end{array}$ \\
\hline pos-clasificar & $\begin{array}{l}\text { Equal variances } \\
\text { assumed } \\
\text { Equal variances } \\
\text { not assumed }\end{array}$ & 55.972 & .000 & $\begin{array}{l}-7.062 \\
-7.062\end{array}$ & $\begin{array}{r}544 \\
489.815\end{array}$ & .000 & $\begin{array}{l}-1.103 \\
-1.103\end{array}$ & $\begin{array}{l}.156 \\
.156\end{array}$ & $\begin{array}{l}-1.409 \\
-1.409\end{array}$ & $\begin{array}{l}-.796 \\
-.796\end{array}$ \\
\hline
\end{tabular}




\section{Análisis considerando el pretest:}

De acuerdo a la Tabla 3 que presenta los resultados en la etapa pretest, el aprendizaje metacognitivo de las cinco habilidades, al analizar la prueba de Levene, nos permite no rechazar la hipótesis nula y asumir la igualdad de varianzas. Si aceptamos lo anterior tenemos, para cada una de las habilidades, los resultados de la prueba t que nos permiten no rechazar la hipótesis nula y concluimos que no existen diferencias significativas entre los grupos control y experimental, atendiendo al aprendizaje metacognitivo de las habilidades señaladas en este estudio.

\section{Análisis considerando el postest:}

De acuerdo a la Tabla 3, que presenta resultados del aprendizaje metacognitivo de las habilidades en el postest, considerando la prueba de Levene, podemos rechazar la hipótesis nula y asumir la desigualdad de varianzas. Aceptando lo anterior y considerando los resultados de la prueba $t$, para las cinco habilidades señaladas, no se acepta la hipótesis nula y se concluye que existen diferencias significativas entre los grupos control y experimental, atendiendo al aprendizaje metacognitivo de las habilidades ya referidas.

Además, se realizó un estudio de varianza unifactorial que se presenta a continuación: 
Tabla 4. Descriptivos de las diferencias en las variables.

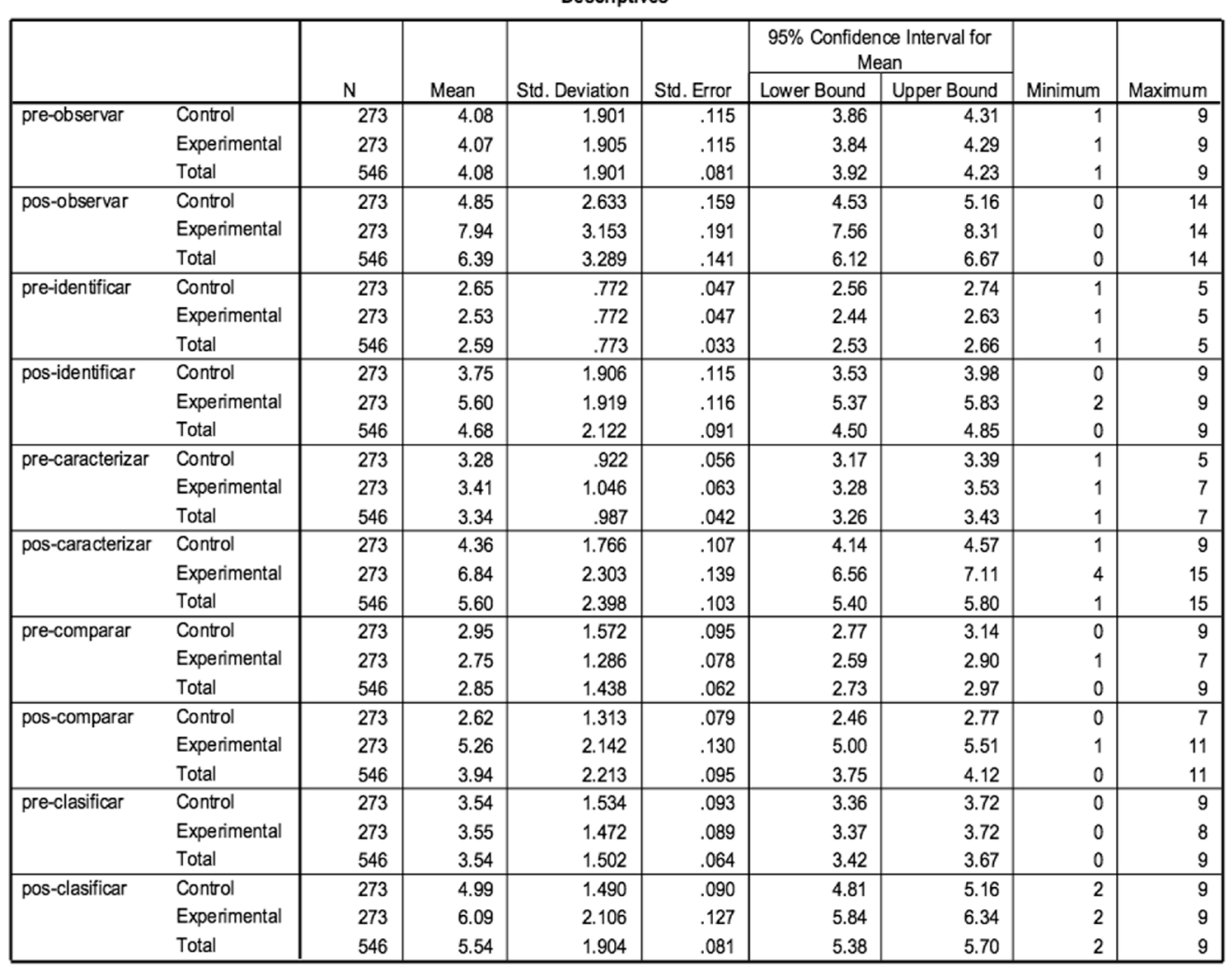

Se puede observar en las muestras independientes, que no existen diferencias significativas. 
Tabla 5. ANOVA para las puntuaciones del cambio.

ANOVA

\begin{tabular}{|c|c|c|c|c|c|c|}
\hline & & $\begin{array}{c}\text { Sum of } \\
\text { Squares }\end{array}$ & df & Mean Square & $\mathrm{F}$ & Sig. \\
\hline \multirow[t]{3}{*}{ pre-observar } & Between Groups & .046 & 1 & .046 & .013 & .911 \\
\hline & Within Groups & 1969.875 & 544 & 3.621 & & \\
\hline & Total & 1969.921 & 545 & & & \\
\hline \multirow[t]{3}{*}{ pos-observar } & Between Groups & 1304.645 & 1 & 1304.645 & 154.642 & .000 \\
\hline & Within Groups & 4589.480 & 544 & 8.437 & & \\
\hline & Total & 5894.125 & 545 & & & \\
\hline \multirow[t]{3}{*}{ pre-identificar } & Between Groups & 1.760 & 1 & 1.760 & 2.954 & .086 \\
\hline & Within Groups & 324.161 & 544 & .596 & & \\
\hline & Total & 325.921 & 545 & & & \\
\hline \multirow[t]{3}{*}{ pos-identificar } & Between Groups & 463.386 & 1 & 463.386 & 126.660 & .000 \\
\hline & Within Groups & 1990.234 & 544 & 3.659 & & \\
\hline & Total & 2453.621 & 545 & & & \\
\hline \multirow[t]{3}{*}{ pre-caracterizar } & Between Groups & 2.117 & 1 & 2.117 & 2.177 & .141 \\
\hline & Within Groups & 529.150 & 544 & .973 & & \\
\hline & Total & 531.267 & 545 & & & \\
\hline \multirow[t]{3}{*}{ pos-caracterizar } & Between Groups & 841.912 & 1 & 841.912 & 199.874 & .000 \\
\hline & Within Groups & 2291.443 & 544 & 4.212 & & \\
\hline & Total & 3133.355 & 545 & & & \\
\hline \multirow[t]{3}{*}{ pre-comparar } & Between Groups & 5.744 & 1 & 5.744 & 2.785 & .096 \\
\hline & Within Groups & 1121.941 & 544 & 2.062 & & \\
\hline & Total & 1127.685 & 545 & & & \\
\hline \multirow[t]{3}{*}{ pos-comparar } & Between Groups & 952.090 & 1 & 952.090 & 301.711 & .000 \\
\hline & Within Groups & 1716.667 & 544 & 3.156 & & \\
\hline & Total & 2668.756 & 545 & & & \\
\hline \multirow[t]{3}{*}{ pre-clasificar } & Between Groups & .016 & 1 & .016 & .007 & .932 \\
\hline & Within Groups & 1229.429 & 544 & 2.260 & & \\
\hline & Total & 1229.445 & 545 & & & \\
\hline \multirow[t]{3}{*}{ pos-clasificar } & Between Groups & 165.936 & 1 & 165.936 & 49.877 & .000 \\
\hline & Within Groups & 1809.832 & 544 & 3.327 & & \\
\hline & Total & 1975.767 & 545 & & & \\
\hline
\end{tabular}


Al considerar el análisis de varianza unifactorial se concluye que en todos los aspectos referentes al aprendizaje metacognitivo de las habilidades —observar, identificar, caracterizar, comparar y clasificar-, en la etapa pretest no se detectaron diferencias significativas entre los grupos control y experimental; pero, en la etapa postest, sí las hubo.

A continuación se presenta un resumen del resultado obtenido al realizar el análisis de covarianza:

Tabla 6. Resumen del análisis de covarianza.

\begin{tabular}{|l|l|}
\hline \multicolumn{1}{|c|}{ ASPECTOS } & \multicolumn{1}{c|}{ Influencia del Pretest } \\
\cline { 2 - 2 } & No ha influido significativamente \\
\hline $\begin{array}{l}\text { Aprendizaje metacognitivo } \\
\text { de la habilidad observar }\end{array}$ & No ha influido significativamente \\
\hline $\begin{array}{l}\text { Aprendizaje metacognitivo } \\
\text { de la habilidad identificar }\end{array}$ & No ha influido significativamente \\
\hline $\begin{array}{l}\text { Aprendizaje metacognitivo } \\
\text { de la habilidad caracterizar }\end{array}$ & No ha influido significativamente \\
\hline $\begin{array}{l}\text { Aprendizaje metacognitivo } \\
\text { de la habilidad comparar }\end{array}$ & No ha influido significativamente \\
\hline $\begin{array}{l}\text { Aprendizaje metacognitivo } \\
\text { de la habilidad clasificar }\end{array}$ & \\
\hline
\end{tabular}

Al considerar el análisis de covarianza se aprecia la no influencia significativa sobre la manipulación experimental del aprendizaje metacognitivo de las habilidades: observar, identificar, caracterizar, comparar y clasificar del pretest.

Lo anterior nos permite considerar, en términos reservados, un acercamiento a la validación de la propuesta de aprendizaje metacognitivo de las habilidades mencionadas. 


\section{ÍNDICE DE DESEMPEÑO DEL DOCENTE EN LOS GRUPOS CONTROL Y EXPERIMENTAL}
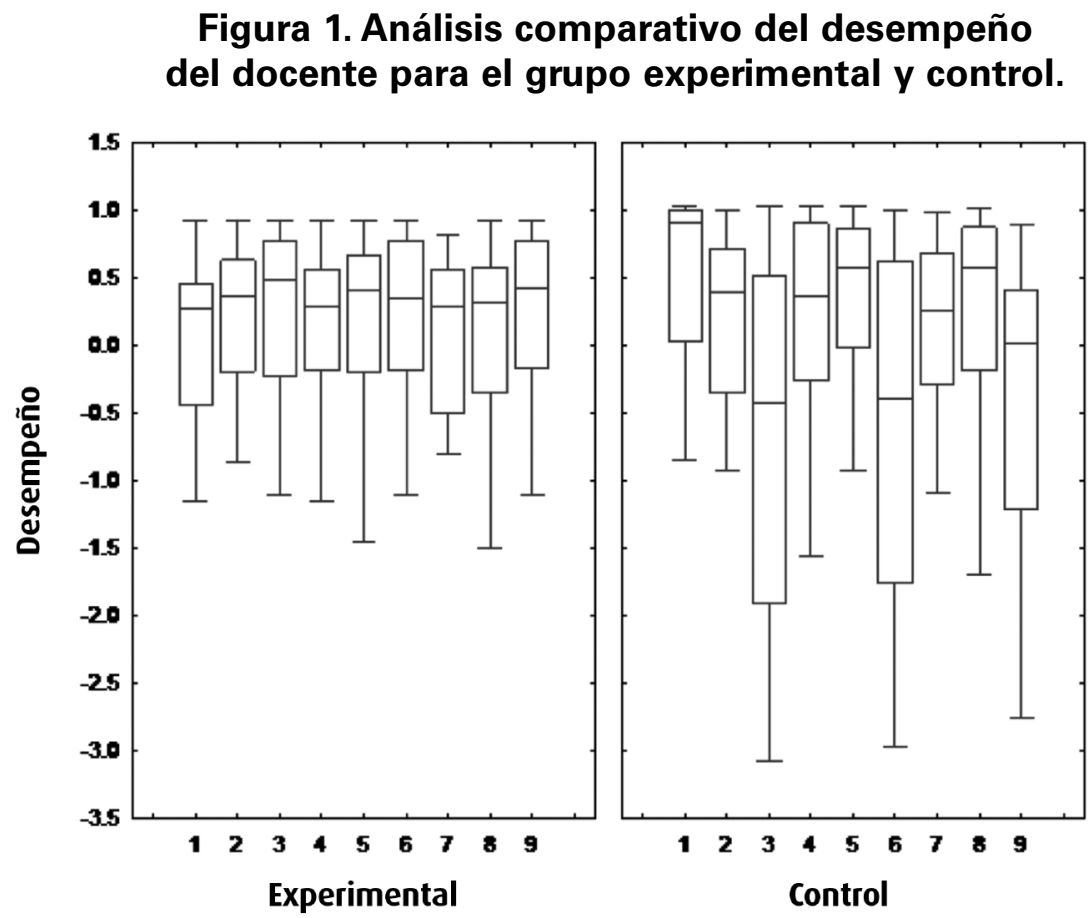

En esta gráfica se aprecia que, en su mayoría, los docentes del grupo experimental obtuvieron mejores evaluaciones por los estudiantes de ese grupo. Situación opuesta a las evaluaciones de los estudiantes del grupo control.

Se aprecia, en los datos del desempeño docente recogidos con la escala, que los resultados son significativos y permiten valorar la importancia que cobra la motivación de los profesores ante un proceso de seguimiento específico, donde él, como agente principal del 
mismo, debe demostrar el aprendizaje metacognitivo de habilidades a partir de su enseñanza a los estudiantes.

\section{RESULTADOS DEL ESTUDIO CUALITATIVO}

Este estudio se realizó través de entrevistas en profundidad, tomando en cuenta la metodología propuesta por Miguel Martínez Miguéles (2006). La categorización y la estructuración, fueron realizadas manualmente. La indagación acerca de la perspectiva didáctica que tienen los profesores que participaron en el estudio, se llevó a cabo al considerar ocho entrevistas semiestructuradas a igual número de formadores de docentes, con una duración promedio de una hora 30 minutos, sin rigidez alguna. Cuatro de ellos participaron en el grupo control y otros cuatro en el experimental. De estos ocho entrevistados, tres tomaron el curso de preparación para la intervención y cinco no lo hicieron.

La estrategia seguida puede sintetizarse en: pregunta generadora, dimensión de análisis, escenario básico de indagación, determinación de los actores e informantes claves, determinación de técnicas e instrumentos y análisis e interpretación de la información. En cuanto a los resultados, se advierte que existe resistencia de los profesores al considerar intrascendente la vinculación teórico-práctica en el desempeño docente. Tres docentes del grupo control, perciben que la forma de enseñar se logra solo con la práctica. Además, conciben que los estudiantes retomen su forma de enseñar de otros docentes que han impactado en su forma de aprender.

Los informantes claves perciben que los planes de estudio propician incomunicación y confusión entre los profesores, porque los programas plantean orientaciones pedagógicas con un lenguaje poco claro. Precisan que ante ello, cada profesor supone un proceso educativo alternativo, que la mayoría de las veces no corresponde con el Plan y programas de estudio; sin embargo, es aplicado para el desarrollo de competencias docentes en los futuros educadores de educación primaria. 
Se pudo apreciar que existen algunos docentes que poseen actitudes y aptitudes de percibir al proceso didáctico como un sistema, y se esfuerzan por revisar documentos que les permitan obtener claridad en sus concepciones de enseñanza aprendizaje. No obstante, la frágil visión de la gestión didáctica que a veces tienen como base los directivos, algunos docentes de formadores y alumnos, desautorizan a tales profesores con ideas progresistas en búsqueda de mejora y transformación de su quehacer didáctico.

La perspectiva de enseñanza y aprendizaje de los docentes formadores de profesores de educación primaria, visto a través de ocho observadores con vivencias y experiencias diferentes - como se sintetiza en este apartado y que obedece a razones propias de la publicación del presente trabajo-, puede concretarse en los siguientes términos: los formadores de docentes no han logrado todavía la apropiación didáctica suficiente del proceso enseñanza aprendizaje, con su carácter de sistema, para lograr planearlo, ejecutarlo y evaluarlo; la naturaleza del Plan de estudios 1997 y los programas curriculares que emanan del mismo parecen suponer ese dominio de competencias didácticas por parte del formador de docentes. Por tanto, es fácil comprender que el aprendizaje metacognitivo de habilidades tenga dificultades al enseñarse y al aprenderse.

\section{CONCLUSIONES}

Este trabajo surge a partir de la necesidad que presenta el profesorado de una cultura académica y un desarrollo profesional sólidos. Es trascendental alcanzar lo anterior, para arribar al desarrollo de una educación de calidad en nuestro estado y país. En la literatura revisada se plantea la necesidad de que este aprendizaje sea metacognitivo, es decir, que se logre con una correcta flexibilidad, autorregulación y solidez (Perrenoud, 2008; Tobón, 2006; Moltó, 2001; Zilberstein, Portela y Mc. Pherson, 1999).

En la evaluación al desempeño docente se percibe que los estudiantes son capaces de evidenciar el proceso educativo con el que 
interactuaron los docentes con ellos. En este, los profesores destacan una perspectiva del proceso de enseñanza aprendizaje que no tiene relación directa con el enfoque constructivista del proceso educativo (Perrenoud, 2004; Moltó, 2001; Aebli, 2001; Bermúdez y Rodríguez, 1996; Ausubel, Novak, y Hanesian, 1983; Talizina, 1988).

También se explica la validación de la estrategia y el enfoque utilizado. Esta destaca que la estrategia planteada:

a) Ayuda al desarrollo de un aprendizaje flexible por parte de los estudiantes;

b) contribuye al desarrollo de un aprendizaje sólido de los estudiantes;

c) mejora la autorregulación del aprendizaje de esas habilidades por parte de los estudiantes, $y$

d) contribuye a una mejor dirección de un aprendizaje metacognitivo de esas habilidades por parte de los estudiantes.

Aunque fue necesario en el trabajo utilizar un diseño de grupo no equivalente, los resultados que se muestran tienen implicaciones educativas importantes, que se plantean a continuación:

La estrategia anima al profesorado normalista a adoptarla e integrar en sus actividades de enseñanza el desarrollo del aprendizaje metacognitivo de habilidades explícitamente, y no de la forma convencional como lo hemos venido intentando.

El proceso de enseñanza aprendizaje y su carácter de sistema es un planteamiento importante, que debe destacarse en la formación de los estudiantes de la Licenciatura de Educación Primaria, y que los resultados de la validación muestran como aceptable, dadas las características del diseño de los grupos estudiados, pero son estos mismos los que proveen de argumentos valiosos contra la visión que impera hoy en la formación de docentes, por parte de algunos profesores formadores de docentes que señalan que no es necesario el desarrollo de un aprendizaje metacognitivo. Esos datos obtenidos en esta intervención permiten vislumbrar desde la educación normal, que enseñar a pensar integrando las actividades de pensamiento con los temas que incluyen las demás asignaturas curriculares que cursa el estudiante de la Licenciatura de Educación Primaria, mejora en lugar de empeorar. 
Una oposición común a enseñar a aprender metacognitivamente habilidades se debe a la creencia, entre los docentes, de que no es apropiado para todos los estudiantes. Algunos estudios muestran que muchos profesores creen que el objetivo de enseñar a pensar es apropiado solamente para los estudiantes de alto rendimiento, y no lo es para los estudiantes de bajo rendimiento que están teniendo dificultades para aprender, incluso, hechos básicos (Zohar, 2006),

Al realizar una valoración integral del estudio realizado se puede apreciar lo siguiente:

- El logro del primer objetivo permite concluir que se ha diseñado una estrategia de enseñanza aprendizaje metacognitivo de habilidades que pudiera ser utilizada en la formación de los Licenciados en Educación Primaria, que estudian en escuelas normales, privadas y en zonas urbanas.

- El logro del segundo objetivo permite concluir la realización de un primer acercamiento a la validación de la estrategia diseñada, al lograr la realización de la intervención mediante EEAMPP, que objetiva los planteamientos teóricos señalados y permite obtener datos mediante análisis estadísticos pertinentes.

- En cuanto a la evaluación del desempeño docente, se presentan evidencias que advierten resultados significativamente superiores en los profesores que recibieron la capacitación basada en EEAMHPP y que participaron en la intervención con el grupo experimental, con respecto a los profesores que no fueron capacitados con EEAMHPP, que siguieron trabajando con el enfoque tradicional y que fueron participantes en el grupo control.

- Gracias a las indagaciones de las perspectivas acerca de la enseñanza y el aprendizaje — que sustentan la práctica docente de un grupo de profesores formadores de docentes de la Licenciatura en Educación Primaria de escuelas normales privadas del Estado de Veracruz-, y el planteamiento de los mismos acerca de sus creencias del proceso de enseñanza aprendizaje, se pudo analizar su 
perspectiva educativa e interpretar la postura que subyace mayoritariamente en una práctica docente basada en el sentido común.

A continuación se destacan las limitaciones que pueden advertirse en el presente estudio, y que probablemente han podido incidir en los resultados:

- El tamaño de la muestra, tanto de profesores y alumnos, no permite la generalización de los resultados.

- Es perceptible que la selección de los alumnos de segundo semestre fue realizada de forma no aleatoria, y aunque al asignar estos a los grupos control y experimental se hizo al azar, los docentes formadores de profesores fueron asignados por conveniencia.

- El tipo de diseño elegido se considera el idóneo; indiscutiblemente se hubiera mejorado con un mayor número de profesores y alumnos en ambos grupos de estudio, elegidos aleatoriamente.

- Existen factores que pudieron haber incidido en los resultados obtenidos con la estrategia de enseñanza y aprendizaje metacognitivo de habilidades, dado que existieron elementos difíciles de controlar y que fueron perceptibles tales como: la contingencia sanitaria resultante en el país al realizarse la intervención; además de aspectos socioeconómicos, actitudes y aptitudes personales.

- Las limitaciones de una investigación cuasi experimental en el ámbito educativo, particularmente teniendo como objetivos la validación externa como el presente estudio, proceden prioritariamente del control de variables, aspecto que se observa en esta investigación. 


\section{REFERENCIAS BIBLIOGRÁFICAS}

AEBLI, H., Doce formas básicas de enseñar, Narcea, Madrid, España, 2001.

AUSUBEL, D.; NOVAK, J. y HANESIAN, H., Psicología Educativa. Un punto de vista cognoscitivo, Trillas, México, 1976.

BANCO INTERAMERICANO DE DESARROLLO, La política de las políticas públicas. Progreso económico y social en América Latina, Informe 2006, David Rockefeller Center for Latin American Studies Harvard University, Planeta, México, 2006.

BANDURA, A., Auto-eficacia: Cómo afrontamos los cambios de la sociedad actual, Desclée De Brouwer Biblioteca de Psicología, Bilbao, España, 1999.

BERMÚDEZ, M., Bosquejos de Educación para el Pueblo: Ignacio Ramírez e Ignacio Manuel Altamirano. (antología), SEPEl Caballito, Distrito Federal, México, 1985.

BRUNER, J., La importancia de la educación, Paidos Educador, Barcelona, España, 1990.

CASTELLANOS, D., Aprender y enseñar en la escuela. Ed. Pueblo y Educación, La Habana. 2002.

CENEVAL, Resultados del Examen General de Conocimientos de la Licenciatura en Educación Primaria, Datos en bruto no publicados, México, 2005.

COLL, C., Aprendizaje escolar y construcción del conocimiento, Paidos, Madrid, 1990.

DANILOV, M. y SKATKIN, M., Didáctica de la Escuela Media, La Habana: Libros para la Educación, 1980.

DE LA ORDEN, A., Evaluación de los Efectos de los Programas de Intervención. Antología del Seminario de Evaluación Educativa. Doctorado en educación: diagnóstico, medida y evaluación de la intervención educativa, Universidad Anáhuac, México, 2006. 
DÍAZ BARRIGA, A. Didáctica y currículum, Paidós, Distrito Federal, México, 1997.

DIRECCIÓN GENERAL DE EDUCACIÓN NORMAL Y SUPERIOR, Boletín Informativo, Veracruz, México, 2007.

FLAVELL, J.H., «Metacognitive development», en J.M. Scandura \& C.J. Brainerd (eds.), Structural/process models of complex human behavior, Sifthoff \& Noordhoff, Netherlands, 1978.

GIMENO, J y PÉREZ, A., Comprender y transformar la enseñanza, Morata, Madrid, España, 1992.

GUTIÉRREZ, V. y MOLTÓ, E., Metodología para la enseñanza de los conocimientos, las cadenas verbales y los procedimientos, Escuela Normal La Paz de Veracruz, Veracruz, México, 2004.

, «Método para la enseñanza y el aprendizaje metacognitivo de habilidades algorítmicas», en VVAA, Proceso de enseñanza aprendizaje: bases neurales y contexto sociocultural, Ceguro Editores, Lima, Perú, 2007.

JACKSON, Ph., La vida en las aulas, Morata, España, 2000.

MOLTÓ, E., Un enfoque para la educación en ciencias, Universidad Veracruzana, Veracruz, México, 2001.

PERRENOUD, P., Diez nuevas competencias para enseñar, SEP, México, 2004a.

, Desarrollar la práctica reflexiva en el oficio de enseñar. Crítica y fundamentos 1, Graó, Barcelona, España, 2004b.

PIAGET, J., El nacimiento de la inteligencia en el niño, Grijalbo, México, 1977.

PIMIENTA, J., Diseño y validación de un modelo didáctico para la educación media superior, Tesis de doctorado, Escuela de Educación, Universidad Anáhuac, Estado de México, México, 2006.

, Las competencias en la docencia universitaria.

Preguntas frecuentes, Pearson-Prentice Hall, Estado de México, México, 2011. 
TOBÓN, S.; PIMIENTA, J.; GARCÍA; J., Secuencias didácticas: aprendizaje y evaluación de competencias. Pearson Educación, México, 2010.

POZO, J., Aprendices y maestros. Una nueva cultura del aprendizaje, Alianza Editorial, Madrid, 1998.

RODRÍGUEZ M. y BERMÚDEZ, R., Teoría y metodología del aprendizaje, Pueblo y Educación, La Habana, Cuba, 1996.

SAINT-ONGE, M., Yo explico, pero ellos... ¿aprenden?, Mensajero, Bilbao, España, 1997.

SEOCK-HO, K., CIA: A Computer Program for Classical Item Analisis, Universidad de Georgia, Estados Unidos de América, 1999.

SEP, Licenciatura en Educación Primaria. Plan de Estudios 1997. Programa para la Transformación y Fortalecimiento Académico de las Escuelas Normales. Educativa, México, 1997.

SEP, Prioridades y Retos de la Educación Básica. Curso Básico de Formación Continu, Dirección General de Formación Continua de Maestros en Servicio. Ciclo Escolar 2008-2009, México, 2008.

TALIZINA, N., Psicología de la enseñanza, Progreso, 1998.

TOBÓN, Sergio (2006). Competencias en la Educación Superior. Colombia: ECOE Ediciones, 2006.

VYGOTSKY, L., Pensamiento y lenguaje, Quinto Sol, México, 1998.

ZILBERSTEIN, J.; PORTELA, R. y MCPHERSON, M., Didáctica integradora vs didáctica tradicional, Pueblo y Educación, La Habana, Cuba, 1999.

ZOHAR, A., El pensamiento de orden superior en las clases de ciencias: objetivos, medios y resultados de investigación, Enseñanza de las Ciencias, 24(2), 157-172, 2006. 